\title{
Temperature dependence of ultraviolet line parameters in network and internetwork regions of the quiet Sun and coronal holes
}

\author{
X. Wang ${ }^{1,2}$, S. W. McIntosh ${ }^{2}$, W. Curdt ${ }^{3}$, H. Tian ${ }^{4}$, H. Peter ${ }^{3}$, and L.-D. Xia ${ }^{5}$ \\ 1 School of Earth and Space Sciences, Peking University, 100871 Beijing, PR China \\ e-mail: wangxinpku0209@gmail.com \\ 2 High Altitude Observatory, National Center for Atmospheric Research, PO Box 3000, Boulder, CO 80307, USA \\ 3 Max-Planck-Institut für Sonnensystemforschung, 37191 Katlenburg-Lindau, Germany \\ ${ }^{4}$ Harvard-Smithsonian Center for Astrophysics, 60 Garden Street, Cambridge, MA 02138, USA \\ 5 School of Space Science and Physics, Shandong University at Weihai, 264209 Weihai, PR China
}

Received 10 August 2012 / Accepted 22 July 2013

\begin{abstract}
Aims. We study the temperature dependence of the average Doppler shift and the non-thermal line width in network and internetwork regions for both the quiet Sun $(\mathrm{QS})$ and the coronal hole $(\mathrm{CH})$, by using observations of the Solar Ultraviolet Measurements of Emitted Radiation instrument onboard the Solar and Heliospheric Observatory spacecraft.

Methods. We obtain the average Doppler shift and non-thermal line width in the network regions of QS, internetwork regions of QS, network regions of $\mathrm{CH}$, and internetwork regions of $\mathrm{CH}$ by applying a single-Gaussian fit to the line profiles averaged in each of the four regions. The formation temperatures of the lines we use cover the range from $10^{4}$ to $1.2 \times 10^{6} \mathrm{~K}$. Two simple scenarios are proposed to explain the temperature dependence of the line parameters in the network regions. In one of the scenarios, the spectral line consists of three components: a rapid, weak upflow generated in the lower atmosphere, a nearly static background, and a slow cooling downflow. In the other scenario, there are just two components, which include a bright core component and a faint wide tail one.

Results. An enhancement of the Doppler shift magnitude and the non-thermal line width in network regions compared to the internetwork regions is reported. We also report that most transition region lines are less redshifted (by $0-8 \mathrm{~km} \mathrm{~s}^{-1}$ ) and broader (by $0-5 \mathrm{~km} \mathrm{~s}^{-1}$ ) in $\mathrm{CH}$ compared to the counterparts of QS. In internetwork regions, the difference in the Doppler shifts between the coronal hole and the QS is slightly smaller, especially for the lines with formation temperatures lower than $2 \times 10^{5} \mathrm{~K}$. And the two simple scenarios can reproduce the variation in the line parameters with the temperature very well.

Conclusions. Our results suggest that the physical processes in network and internetwork regions are different and that one needs to separate network and internetwork when discussing dynamics and physical properties of the solar atmosphere. The agreement between the results of the observation and our scenarios suggests that the temperature dependence of Doppler shifts and line widths might be caused by the different relative contributions of the three components at different temperatures. The results may shed new light on our understanding of the complex chromosphere-corona mass cycle. However, the existing observational results do not allow us to distinguish between the two scenarios. At this stage, a high-resolution instrument Interface Region Imaging Spectrograph is highly desirable.
\end{abstract}

Key words. Sun: atmosphere - Sun: UV radiation - line: profiles

\section{Introduction}

One of the important problems related to the mass cycle and energy balance of the outer solar atmosphere in solar physics has been to understand the prevailing redshift of the UV lines formed in the solar transition region (TR) since their discovery (Doschek et al. 1976). The temperature dependence of the average Doppler shift and non-thermal width in solar TR and corona has been investigated by solar ultraviolet spectroscopic observations in previous studies (e.g., Brekke et al. 1997; Chae et al. 1998a,b; Peter 1999; Peter \& Judge 1999; Teriaca et al. 1999; Xia et al. 2004; Tian et al. 2010; Dadashi et al. 2011). The results show that the TR lines are redshifted with the average Doppler shifts reaching a maximum of about 14,10 , and $7 \mathrm{~km} \mathrm{~s}^{-1}$ in active regions (AR), quiet Sun (QS), and coronal holes $(\mathrm{CH})$, respectively, at $T \approx 2 \times 10^{5} \mathrm{~K}$. At higher temperatures, above $10^{6} \mathrm{~K}$, the net line shift turns into a blueshift (Peter 1999; Peter \& Judge 1999).
This is the case even in the QS, where the net shift drops to zero well above $10^{6} \mathrm{~K}$ (Dadashi et al. 2011).

A variety of physical processes have been proposed to explain the observed redshift. One of the ideas is that it is caused by the return of the previously heated spicular material, which was first proposed by Pneuman \& Kopp (1987) and has been verified by recent work (McIntosh et al. 2012) through both imaging and spectroscopic studies. Another idea suggested by $\mathrm{Tu}$ et al. (2005a,b) and He et al. (2008) is that the redshift is associated with the downflows produced by the magnetic reconnection between the open funnels and the adjacent closed loops at the height of about $5 \mathrm{Mm}$ above the photosphere. Moreover, Hansteen (1993) argued that the nanoflare induced downwardpropagating waves generated in the corona could also produce the redshift in TR lines. The blueshift of the coronal line is often considered as a possible signature of the nascent solar wind 
(Hassler et al. 1999; Peter \& Judge 1999; Wilhelm et al. 2000; Xia et al. 2003; Tian et al. 2010; Tu et al. 2005a,b; Wiegelmann et al. 2005). The temperature dependence of the average nonthermal width derived from a single-Gaussian fit has a very similar trend to that of the average Doppler shift. It increases with temperature and peaks nearly at the same temperature as the Doppler shift, and then begins to decrease at the coronal temperature. It is also found that the line widths of the line profiles in CHs are somewhat larger than those in QS (Lemaire et al. 1999; Stuki et al. 2000; McIntosh et al. 2007).

Numerical models of the solar atmosphere have also been built to understand the observed phenomena. These models are able to match the observed temperature variations of the average Doppler shift and non-thermal line width very well. For example, Peter et al. $(2004,2006)$ described a three-dimensional MHD forward model spanning the photosphere to the corona, in which the persistent redshifts in the TR lines are caused by the flows induced by the heating through braiding of magnetic flux. Hansteen et al. (2010) also showed some three-dimensional MHD models, while in these models the downflows in TR and the slight upflows in the low corona can be naturally produced by the rapid and episodic heating of the upper chromospheric plasma to coronal temperatures. The heating used in all these $3 \mathrm{D}$ models is related to the stressing of the magnetic field by photospheric and convection zone dynamics, but also in some models is generated by the injection of emerging magnetic flux.

Recent research has revealed the ubiquitous presence of blueward asymmetries of TR and coronal emission line profiles, which is most prominent in loop footpoint regions (e.g., De Pontieu et al. 2009; De Pontieu \& McIntosh 2010; McIntosh \& De Pontieu 2009a,b; McIntosh et al. 2010, 2011, 2012; Tian et al. 2011a,b). Owing to a highly blueshifted emission component, the enhancement in the blue wings was considered to result from the rapid upflows $\left(50-100 \mathrm{~km} \mathrm{~s}^{-1}\right)$ injected from the chromosphere into the corona in association with the so-called type-II spicules (De Pontieu et al. 2007). In addition, McIntosh et al. (2012) shows there are both the downward and upward motions at the atmosphere with low temperature (lower than the "typical" coronal temperature of $1 \mathrm{MK}$ ) through a combination of imaging and spectroscopic observations. The downward flows are reported to have a speed of $\sim 14 \mathrm{~km} \mathrm{~s}^{-1}$, and upward flows have a speed of $\sim 96 \mathrm{~km} \mathrm{~s}^{-1}$. The slow downward flows are considered as the return of the previously heated and inserted materials into the corona and were a natural consequence of the mass cycling between the chromosphere and corona. Accordingly, we present the conjecture that there must be at least three components, which include the rapid upflow, the slow downflow, and a nearly static background, in the profiles of these relatively cool subcoronal emission lines.

It has been suggested that network and internetwork regions have different properties (e.g., Hassler et al. 1999; Peter 2000; Yang et al. 2009; Zhang et al. 1999). Peter (2000) investigated line parameters and also the non-symmetric nature of the line profiles through double-Gaussian fits. This showed that in the internetwork the profiles are single-Gaussian, while the network transition region emission shows an excess in the wings that can be accounted for by a second broad-Gaussian component. However, in the previous studies about the temperature dependence of the line parameters, people hardly differentiated the network regions from the internetwork. Using observations of the Solar Ultraviolet Measurements of Emitted Radiation instrument (SUMER, Wilhelm et al. 1995, 1997; Lemaire et al. 1997) onboard the Solar and Heliospheric Observatory (SOHO) spacecraft, we distinguish network from internetwork and explore the temperature variation of the average Doppler shift and nonthermal line width in network and internetwork regions separately. A simple three-component toy model, using the components discussed above, is built to explain the observed variation in the line parameters. Moreover, a more contemporary twocomponent scenario Peter (2001), including a bright core component related to the small loops and a faint wide tail component emitted from the large coronal funnels, will also be discussed. Our objective in this work is to establish groundwork for the Interface Region Imaging Spectrograph (IRIS).

\section{Observations and data analysis}

The UV spectral data in QS and $\mathrm{CH}$ analyzed here were acquired by SUMER near the solar disk center during the period from 1999 March 8 to March 13. The detector A and slit 2 with a size of $1^{\prime \prime} \times 300^{\prime \prime}$ were used. During the interval, SUMER worked in both the raster scan and reference spectrum modes. The reference spectrum mode refers to an observing sequence that consists of a set of about 60 spectral sections obtained sequentially from $780 \AA$ to $1610 \AA$ (Curdt et al. 2001). The data we use here have been previously presented and analyzed by Xia et al. (2004). The collection of these data is listed in Table 1.

Some standard correcting and calibrating processes have been done to the original data by using the routines in solar software (SSW), including the decompression, dead-time correction, odd/even correction, local gain correction, flatfield correction, and geometric distortion correction.

\subsection{Thermal deformation correction and addition geometrical correction}

Eighteen lines (listed in Tables 1 and 2) with different formation temperatures ranging from $10^{4} \mathrm{~K}$ (chromosphere) to $1.2 \times 10^{6} \mathrm{~K}$ (corona) are chosen to perform our analysis. These lines have been commonly used in some related previous works (Brekke et al. 1997; Chae et al. 1998a,b; Peter 1999; Peter \& Judge 1999; Teriaca et al. 1999; Xia et al. 2004; Tian et al. 2010; Dadashi et al. 2011). Unfortunately the temperature region beyond $\log (T / K) \approx 5.5$ is not well sampled owing to the absence of strong and clean lines in our reference spectra. The original intensity, Doppler shift, and line width maps of them can be obtained by applying the single- (for clean lines) or multi-Gaussian (for blended lines) fit to the line profiles. It appears that there are some vertical and horizontal stripes in the Doppler shift maps due to the thermal (Dammasch et al. 1999) and the residual geometrical (Peter \& Judge 1999) distortion of the instrument. To remove them, an additional line-position correction is performed as follows.

1) For the raster scan-mode observations in QS, we first get the average Doppler shift of each column of a cool (or chromospheric) line in a spectral window, and the smooth of the result is the thermal distortion of this window. Analogously, the smooth of the average Doppler shift of each row in a certain line accounts for the residual geometrical distortion of it. We subtract the thermal distortion from each row and the geometrical distortion from each column of the original Dopplergram to obtain the real Doppler shift map.

2) For the raster scan-mode observations in $\mathrm{CH}$, the method, which is applied in QS, is not feasible. Since there are some large structures in the $\mathrm{CH}$ maps, the average of the Doppler shift must include some real signal, not only the distortion. At this point, if the distortion is simply subtracted from the 
Table 1. SUMER observations of QS and $\mathrm{CH}$.

\begin{tabular}{|c|c|c|c|c|c|c|c|}
\hline & & $\begin{array}{c}\text { Date } \\
03 / 1999\end{array}$ & $\begin{array}{l}\text { Time } \\
\text { UTC }\end{array}$ & $\begin{array}{c}\text { Solar coordinate } \\
X\left({ }^{\prime \prime}\right), Y\left({ }^{\prime \prime}\right)\end{array}$ & $\begin{array}{l}\text { Wavelength } \\
(\AA)\end{array}$ & $\begin{array}{l}\text { Exposure } \\
\text { Time (s) }\end{array}$ & $\begin{array}{c}\text { Steps } \\
\left({ }^{\prime \prime}\right)\end{array}$ \\
\hline \multirow{8}{*}{ QS } & S VI 933 & 11 & $03: 22$ & 0,0 & $905-950$ & 325 & 0 \\
\hline & $\begin{array}{l}\text { O VI 1031, C II 1036, } \\
\text { C II 1037, O VI } 1037\end{array}$ & 11 & 04:07 & 0,0 & $1007-1052$ & 325 & 0 \\
\hline & N v 1238, N v 1242 & 8 & $23: 13-03: 14$ & $64 \mathrm{~W}-44 \mathrm{E}, 120 \mathrm{~N}$ & $1234-1254$ & 150 & 1.13 \\
\hline & ${ }^{*} \operatorname{Mg}$ X 1248 & 11 & $05: 32$ & 0,0 & $1228-1272$ & 325 & 0 \\
\hline & ${ }^{*} \mathrm{O}$ V 1259 & 11 & $05: 43$ & 0,0 & $1253-1297$ & 325 & 0 \\
\hline & O I 1304, O I 1306 & 11 & 06:00 & 0,0 & $1291-1335$ & 325 & 0 \\
\hline & $\begin{array}{c}\text { Si IV 1393, O IV 1401, } \\
\text { Si IV } 1402\end{array}$ & 11 & $06: 29$ & 0,0 & $1379-1423$ & 325 & 0 \\
\hline & $\begin{array}{l}\text { Si II } 1533,{ }^{*} \text { Ne VIII } 1540, \\
\text { C IV } 1548, \text { C IV } 1550\end{array}$ & 8 & $15: 20-19: 20$ & $64 \mathrm{~W}-44 \mathrm{E}, 120 \mathrm{~N}$ & $1532-1552$ & 150 & 1.13 \\
\hline \multirow{8}{*}{$\mathrm{CH}$} & S VI 933 & 13 & 07:06 & $240 \mathrm{~W}, 320 \mathrm{~N}$ & $905-950$ & 325 & 0 \\
\hline & $\begin{array}{l}\text { O VI 1031, C II 1036, } \\
\text { C II } 1037, \text { O VI } 1037\end{array}$ & 13 & 07:51 & $240 \mathrm{~W}, 320 \mathrm{~N}$ & $1007-1052$ & 325 & 0 \\
\hline & N v 1238, N v 1242 & 12 & 21:00-01:01 & $234 \mathrm{~W}-126 \mathrm{~W}, 330 \mathrm{~N}$ & $1234-1254$ & 150 & 1.13 \\
\hline & ${ }^{*} \operatorname{Mg} \times 1248$ & 13 & 09:16 & $240 \mathrm{~W}, 320 \mathrm{~N}$ & $1228-1272$ & 325 & 0 \\
\hline & ${ }^{*} \mathrm{O} v 1259$ & 13 & $09: 27$ & $240 \mathrm{~W}, 320 \mathrm{~N}$ & $1253-1297$ & 325 & 0 \\
\hline & O I 1304, O I 1306 & 13 & 09:44 & $240 \mathrm{~W}, 320 \mathrm{~N}$ & $1291-1335$ & 325 & 0 \\
\hline & $\begin{array}{c}\text { Si IV 1393, O IV 1401, } \\
\text { Si IV } 1402\end{array}$ & 13 & $10: 24$ & $240 \mathrm{~W}, 320 \mathrm{~N}$ & $1380-1423$ & 325 & 0 \\
\hline & $\begin{array}{l}\text { Si II } 1533,{ }^{*} \mathrm{Ne} \text { VIII } 1540, \\
\text { C IV } 1548, \text { C IV } 1550\end{array}$ & 11 & $08: 05-12: 05$ & 141 E-249 E, $290 \mathrm{~N}$ & $1532-1552$ & 150 & 1.13 \\
\hline
\end{tabular}

Notes. The lines marked by * are observed in second order in the SUMER spectrum.

data, some real signal might be removed. Fortunately, the data in $\mathrm{CH}$ we use here are taken using the same slit and the same observation mode as in QS, so what we do here is to use the distortion derived from QS data to correct for the $\mathrm{CH}$ data observed under the same mode for each line. This method is reliable, because for a selected line, these two kinds of distortion are related to the observation mode of the instrument in a short period of time and not the physical effects induced by the Sun.

3) If the observations are made in the reference spectrum and not in raster mode, there is no reliable way to do the thermal correction. In this case, what we do is to ignore the thermal distortion with a magnitude of about $0-2 \mathrm{~km} \mathrm{~s}^{-1}$, and just take the geometrical distortion into account.

Figures 1 and 2 show the maps of the intensity, corrected Doppler shift, and line width in Si II, C IV, N V, and Ne VIII in QS and $\mathrm{CH}$.

\section{2. $\mathrm{CH}$ and network region selection}

In general, $\mathrm{CH}$ boundaries can be defined by the intensity contours of hot TR or coronal lines (like Ne VIII observed in raster scan mode). The intensity of the line $\mathrm{MgX}$ observed in reference spectrum mode is also used to distinguish the QS and $\mathrm{CH}$ by first smoothing the intensity along the slit and then considering the dark segments as $\mathrm{CH}$ areas. In the spectral windows of SUMER with none of these hot lines, the images obtained simultaneously in the Fe XII channel of the Extreme-ultraviolet Imaging Telescope (EIT) on SOHO are used to determine the $\mathrm{CH}$ boundaries.

We use the intensity maps of chromospheric lines or continuum to distinguish between the network and internetwork regions. The data points are first sorted by the radiance of a cool line or a part of continuum in each spectral window, and then $33 \%$ of those points with the highest intensity and $33 \%$ of the points with the lowest intensity are selected as network and internetwork regions, respectively (Deubner \& Fleck 1990; Aiouaz et al. 2005). In the first column of Figs. 1 and 2, the bright and dark contours represent the network and internetwork derived from the intensity maps of Si II, respectively. These contours are also superposed on the maps of C IV and Ne VIII that are in the same SUMER spectral window. The network and internetwork of $\mathrm{N} \mathrm{V}$ are defined by using the intensity of the continuum in its spectral window.

\subsection{Absolute wavelength calibration using reference spectra}

The absolute wavelength calibration (i.e., converting the spectral unit from pixel to angstrom) plays a vital role in the absolute Doppler-shift calculation process. The method we use here is the cool-line method (Feldman et al. 1982; Brekke et al. 1997; Chae et al. 1998b; Peter \& Judge 1999; Davey et al. 2006). These previous studies have illustrated that chromospheric lines have nearly no Doppler shift on average, so they can be used as reference lines to deduce the spectral dispersion of a spectral window by applying a linear fit between the wavelength pixels and rest wavelengths of them. To increase the signal-to-noise ratio, the line profiles averaged over the whole observed regions are used to deduce the dispersion. We ensure that at least two reference lines located close to each hot line are used. The absolute Doppler shift is calculated by comparing the real wavelength of the hot line derived from the linear relationship with the known rest wavelength (Kelly 1987; Kaufman \& Martin 1989).

\subsection{Gaussian fit to the normalized average profiles}

The line parameters, including the intensity, Doppler shift, and line width, can be deduced from a Gaussian fit to the line profile. To obtain the average line parameters in the network and internetwork regions, we first normalize the individual profile 
Table 2. Average line parameters of the observed eighteen lines in the network (Net) and internetwork (Cel) regions of QS and CH.

\begin{tabular}{|c|c|c|c|c|c|c|c|c|c|c|}
\hline & \multirow[b]{2}{*}{ wav. $(\AA)$} & \multirow[b]{2}{*}{$\log (T / \mathrm{K})$} & \multicolumn{4}{|c|}{ Absolute Doppler shift $\left(\mathrm{km} \mathrm{s}^{-1}\right)$} & \multicolumn{4}{|c|}{ Non-thermal line width $\left(\mathrm{km} \mathrm{s}^{-1}\right)$} \\
\hline & & & QS/Cel & QS/Net & $\mathrm{CH} / \mathrm{Cel}$ & $\mathrm{CH} / \mathrm{Net}$ & QS/Cel & QS/Net & $\mathrm{CH} / \mathrm{Cel}$ & $\mathrm{CH} / \mathrm{Net}$ \\
\hline$\overline{\mathrm{O} I}$ & 1304.856 & 4.0 & $0.87 \pm 0.24$ & $1.80 \pm 0.35$ & $1.29 \pm 0.32$ & $1.04 \pm 0.34$ & $21.08 \pm 0.02$ & $22.58 \pm 0.04$ & $22.49 \pm 0.03$ & $24.68 \pm 0.03$ \\
\hline O I & 1306.029 & 4.0 & $0.62 \pm 0.35$ & $0.53 \pm 0.51$ & $0.84 \pm 0.45$ & $1.18 \pm 0.37$ & $16.67 \pm 0.02$ & $18.15 \pm 0.04$ & $18.23 \pm 0.04$ & $21.53 \pm 0.05$ \\
\hline Si II & 1533.432 & 4.1 & $2.17 \pm 1.17$ & $3.46 \pm 1.06$ & $1.84 \pm 1.32$ & $2.89 \pm 1.07$ & $17.65 \pm 1.58$ & $20.88 \pm 1.46$ & $18.87 \pm 1.80$ & $23.90 \pm 1.51$ \\
\hline C II & 1036.337 & 4.4 & $5.72 \pm 0.68$ & $7.10 \pm 1.49$ & $4.10 \pm 2.33$ & $2.69 \pm 2.34$ & $22.11 \pm 0.72$ & $26.20 \pm 1.32$ & $21.00 \pm 1.16$ & $26.29 \pm 1.48$ \\
\hline C II & 1037.018 & 4.4 & $4.70 \pm 1.13$ & $5.79 \pm 2.43$ & $4.10 \pm 1.90$ & $4.13 \pm 1.82$ & $28.15 \pm 2.91$ & $34.03 \pm 3.60$ & $29.20 \pm 3.55$ & $34.14 \pm 4.14$ \\
\hline $\mathrm{Si}$ IV & 1393.755 & 4.9 & $4.18 \pm 0.95$ & $8.22 \pm 1.22$ & $3.47 \pm 1.49$ & $3.36 \pm 1.35$ & $22.16 \pm 0.22$ & $25.52 \pm 0.24$ & $24.86 \pm 0.43$ & $27.22 \pm 0.23$ \\
\hline $\mathrm{Si}$ IV & 1402.770 & 4.9 & $4.23 \pm 1.20$ & $8.48 \pm 1.48$ & $2.17 \pm 5.34$ & $2.35 \pm 2.32$ & $23.34 \pm 1.59$ & $27.94 \pm 1.73$ & $27.15 \pm 2.51$ & $29.46 \pm 1.97$ \\
\hline C IV & 1548.204 & 5.0 & $5.84 \pm 1.96$ & $9.15 \pm 1.48$ & $4.02 \pm 3.27$ & $4.68 \pm 1.82$ & $25.02 \pm 1.76$ & $28.79 \pm 1.13$ & $27.03 \pm 2.43$ & $30.73 \pm 1.36$ \\
\hline C IV & 1550.781 & 5.0 & $6.33 \pm 3.26$ & $8.81 \pm 2.00$ & $5.64 \pm 5.01$ & $4.39 \pm 2.46$ & $25.89 \pm 2.80$ & $27.80 \pm 1.95$ & $29.05 \pm 3.36$ & $29.64 \pm 2.28$ \\
\hline O IV & 1401.156 & 5.2 & $5.65 \pm 1.94$ & $10.19 \pm 2.20$ & $3.63 \pm 3.05$ & $4.91 \pm 2.54$ & $20.75 \pm 2.46$ & $27.35 \pm 1.60$ & $25.91 \pm 2.84$ & $29.81 \pm 1.33$ \\
\hline S VI & 933.380 & 5.3 & $11.87 \pm 1.13$ & $14.26 \pm 0.98$ & $7.85 \pm 1.23$ & $9.06 \pm 1.31$ & $29.45 \pm 1.65$ & $32.01 \pm 0.33$ & $32.43 \pm 0.98$ & $33.54 \pm 0.49$ \\
\hline $\mathrm{N}$ V & 1238.821 & 5.3 & $10.05 \pm 1.39$ & $13.30 \pm 1.57$ & $3.58 \pm 1.98$ & $4.94 \pm 2.27$ & $27.94 \pm 1.84$ & $31.34 \pm 1.86$ & $30.62 \pm 2.77$ & $32.91 \pm 2.93$ \\
\hline N V & 1242.804 & 5.3 & $8.66 \pm 1.87$ & $12.05 \pm 2.00$ & $4.63 \pm 2.79$ & $6.10 \pm 3.05$ & $22.14 \pm 1.96$ & $28.05 \pm 1.78$ & $23.29 \pm 2.50$ & $26.37 \pm 2.28$ \\
\hline $\mathrm{OV}$ & 629.730 & 5.4 & $7.97 \pm 0.70$ & $8.43 \pm 0.91$ & $5.99 \pm 0.88$ & $6.14 \pm 1.14$ & $30.01 \pm 0.10$ & $33.48 \pm 0.05$ & $31.29 \pm 0.11$ & $35.44 \pm 0.10$ \\
\hline O VI & $1031.926^{1}$ & 5.5 & $8.87 \pm 0.20$ & $8.98 \pm 0.29$ & $6.64 \pm 0.37$ & $6.71 \pm 0.50$ & $34.24 \pm 0.70$ & $39.13 \pm 1.21$ & $35.52 \pm 1.25$ & $38.89 \pm 1.76$ \\
\hline O VI & $1037.617^{1}$ & 5.5 & $10.45 \pm 0.84$ & $12.32 \pm 1.24$ & $5.89 \pm 1.41$ & $5.19 \pm 1.92$ & $34.91 \pm 0.07$ & $38.26 \pm 0.09$ & $35.70 \pm 0.13$ & $39.58 \pm 0.16$ \\
\hline $\mathrm{Ne}$ VIII & $770.428^{2}$ & 5.8 & $-1.81 \pm 1.27$ & $-2.64 \pm 1.22$ & $-6.80 \pm 2.74$ & $-9.77 \pm 2.60$ & $24.71 \pm 1.66$ & $25.84 \pm 1.58$ & $29.18 \pm 1.18$ & $30.13 \pm 0.94$ \\
\hline $\operatorname{Mg} X$ & $624.968^{3}$ & 6.1 & $-1.11 \pm 1.23$ & $-1.61 \pm 1.61$ & $-6.59 \pm 1.62$ & $-7.09 \pm 2.34$ & $23.37 \pm 2.38$ & $27.35 \pm 3.75$ & $27.67 \pm 3.27$ & $26.33 \pm 4.90$ \\
\hline
\end{tabular}

Notes. ${ }^{(1)}$ Kaufman \& Martin (1989); ${ }^{(2)}$ Dammasch et al. (1999); Peter (1999); ${ }^{(3)}$ Peter \& Judge (1999). The rest wavelengths of all the other ions are from Kelly (1987).

by its total intensity, and then we get the average of these normalized profiles to the network and internetwork regions of QS and $\mathrm{CH}$ that are fitted afterwards to deduce the Doppler shift and the line width. It is well known that there is a positive correlation between the observed redshift and the intensity of the line profile (Brynildsen et al. 1998; Curdt et al. 2008; Dammasch et al. 2008), so we use the normalized line profiles here to avoid the intense profiles dominating the weak ones that can lead to the averaged profiles being shifted towards the red wing. Another method of performing this step is to calculate the line parameters in every single pixel first, and then get the averaged values of them at different areas of interest. In fact, the results derived from the first method could be effected by the averaging profile process, which may cause an enhancement of the line width, and the second method has the problem of low signal-to-noise at some pixels. The results we present in the following parts are derived from the former one, but we have also calculated the averaged line parameters using the second method, and no significant difference is found between them. We also focus on the non-thermal line width instead of the total line width obtained directly from the Gaussian fit. This means that the thermal line width and the instrumental line width derived from the SSW routine con_width_funct_4.pro are both removed from the total line width. The thermal line width is calculated using the formation temperature related to the temperature of the maximum abundance of the ions in the Mazzotta et al. (1998) ionization equilibrium. Chae et al. (1998b) has pointed out that for the lines used here, the temperature of the maximum abundance is not very different from the effective temperature of the formation of the ions. However, for some other ions, like Fe VIII, these two kinds of temperature are distinct (Brooks et al. 2011), but we do not use it in this work. In addition, our choice is commonly used by other researchers (e.g., Brekke et al. 1997; Chae et al. 1998a,b; Peter 1999; Peter \& Judge 1999; Teriaca et al. 1999; Xia et al. 2004; Tian et al. 2010; Dadashi et al. 2011).
Figure 3 shows the averaged normalized line profiles of Si II, C IV, N V, and Ne VIII. Since C IV and Ne VIII windows show the presence of weaker lines in the red wings, we apply doubleGaussian fit to the profiles of them.

\section{Observational results}

\subsection{Spatial distribution of the line parameters in $\mathrm{QS}$ and $\mathrm{CH}$}

Eighteen lines emitted from different "layers" of the solar atmosphere from chromosphere to corona were chosen for the following analysis. These lines are listed in Table 2, along with the rest wavelengths and temperatures of the maximum abundance of the ions. Six of spectra are observed with rasters, including N v $1238 \AA$, N v $1242 \AA$, Si II $1533 \AA$, Ne VIII $1540 \AA$, C IV $1548 \AA$, and C IV $1550 \AA$, while the rest are from reference spectra. Here we present the maps of N V $1238 \AA$, Si II $1533 \AA$, Ne VIII $1540 \AA$, and C IV $1548 \AA$ taken with rasters in QS and $\mathrm{CH}$ as examples in Figs. 1 and 2, respectively. The non- $\mathrm{CH}$ regions in Fig. 2 are shaded. From the top to the bottom, Figs. 1 and 2 show the maps of intensity, Doppler shift and non-thermal width. The four columns are for QS in Si II, C IV, N V, and Ne VIII, respectively. The bright and dark contours show the network and internetwork boundaries.

From these maps, it is clear that for both QS and CH: (1) the absolute values of Doppler shifts are higher in the network than in the internetwork. It means that for the chromospheric lines and transition region lines, such as Si II, C IV, and N V, the network regions are more redshifted, and that for the coronal lines like Ne VIII, the networks are more blueshifted. For example, in the C IV Dopplergram of QS, the networks at around (20" $\left.125^{\prime \prime}\right)$ and $\left(-5^{\prime \prime}, 60^{\prime \prime}\right)$ enclosed by the green contours are significantly more redshifted than the internetwork patch at $\left(5^{\prime \prime}, 80^{\prime \prime}\right)$ surrounded by the black contours. In the Ne VIII Dopplergram of $\mathrm{CH}$, the network regions located around $\left(-220^{\prime \prime}, 275^{\prime \prime}\right)$ and $\left(-180^{\prime \prime}, 375^{\prime \prime}\right)$ are about $8 \mathrm{~km} \mathrm{~s}^{-1}$ more blueshifted than the 

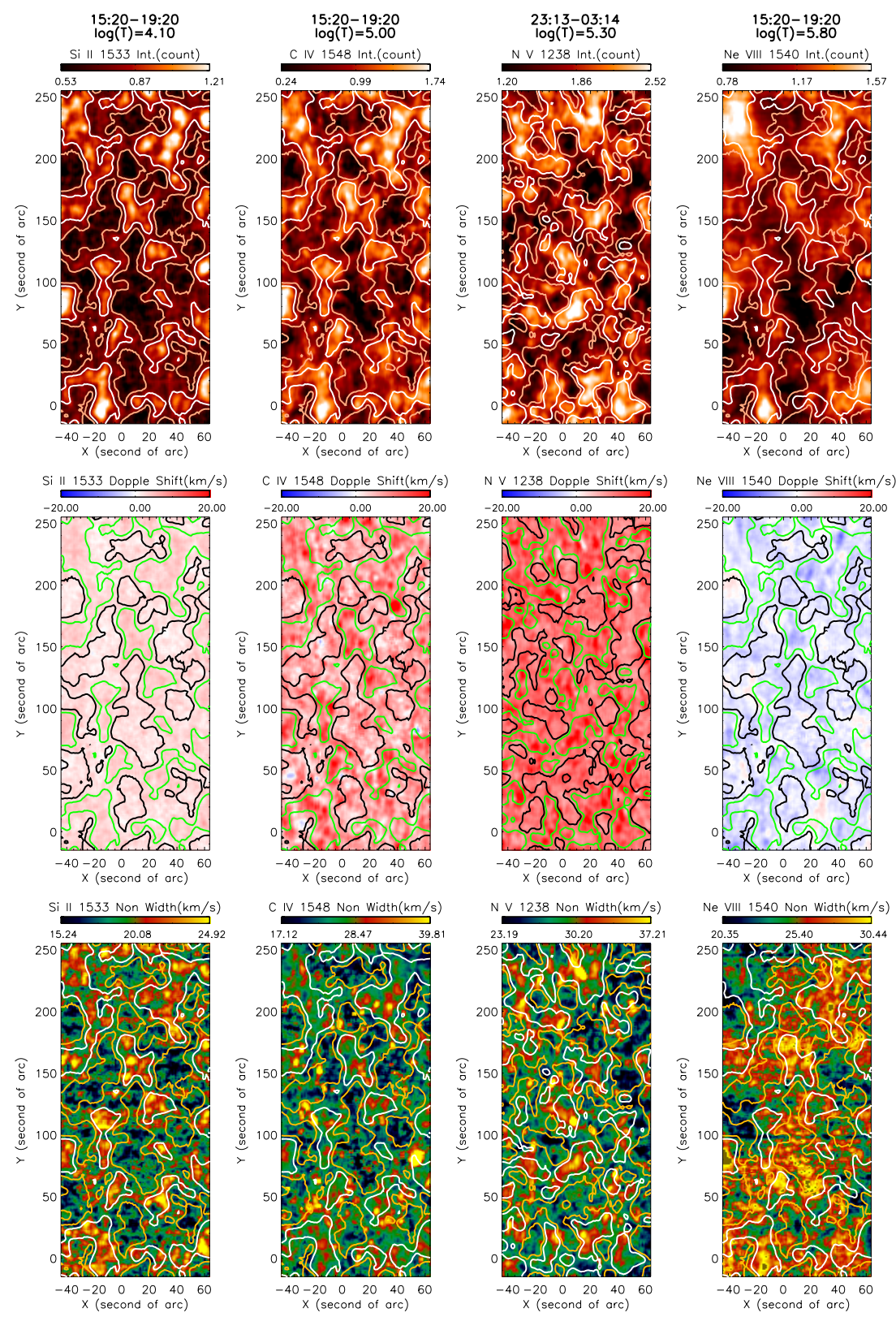

Fig. 1. Spatial distribution of the parameters (line intensity, Doppler shift, and non-thermal width) derived from a Gaussian fit for Si II, C IV, N V, and Ne VIII in QS. The contours represent the network and internetwork regions as seen in the intensity map of Si II or continuum. The network regions are shown as white contours in the intensity and line-width maps, and as the green contours in the Doppler-shift maps. The internetwork regions are shown as brown, black, and orange contours in the maps of intensity, Doppler shift, and line width, respectively.

white internetwork area at $\left(-200^{\prime \prime}, 320^{\prime \prime}\right)$. This result is consistent with Hassler et al. (1999), Xia et al. (2003), and McIntosh et al. (2007), who found that the outflows indicated by the obvious blueshifts occurred predominantly in the network regions. (2) The non-thermal width of the line profiles in network is apparently larger than in internetwork. We can see from the third rows of Figs. 1 and 2 that the red-orange areas corresponding to the broader line profiles are mostly inside the white network contours (except in Ne VIII, because of the low signal-to-noise, especially in $\mathrm{CH}$ ). These phenomena suggest that the main physical processes in the network and internetwork regions are different and that one needs to separate the network and internetwork when discussing the dynamics and physical properties of the quiet solar atmosphere. Next, we are going to study the variance of the average Doppler shift and non-thermal line width as a function of formation temperature in network and internetwork regions separately for both $\mathrm{QS}$ and $\mathrm{CH}$.

\subsection{Average Doppler shifts}

The average line parameters are calculated by applying the Gaussian fit to the average line profiles of the normalized spectra. The results along with the errors of the fits are shown in Fig. 4 and also listed in Table 2. In general, our results show the same trend as previous works (Chae et al. 1998b; Peter \& Judge 1999; Teriaca et al. 1999; Dadashi et al. 2011), which means that the Doppler shifts increase with temperature, reaching a maximum at $\log (T / \mathrm{K}) \approx 5.3$, and then decrease towards higher temperature. Besides, we have some new findings below. 

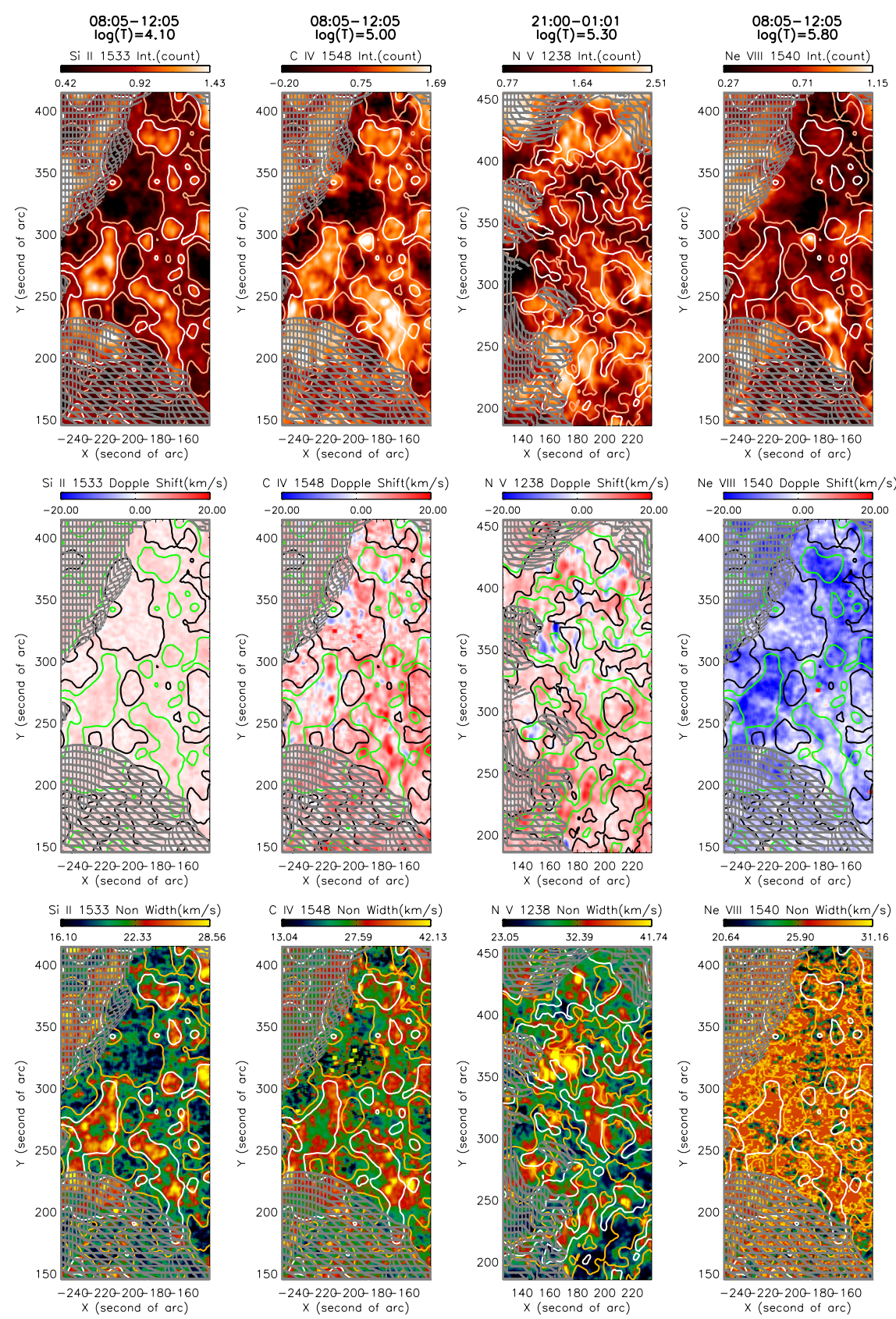

Fig. 2. Spatial distribution of the parameters (line intensity, Doppler shift, and non-thermal width) derived from a Gaussian fit for Si II, C IV, N v, and $\mathrm{Ne}$ VIII in $\mathrm{CH}$ in the same manner as Fig. 1. The non- $\mathrm{CH}$ areas are marked by the shadow.

The bottom lefthand panel of Fig. 4 presents the differences in the Doppler shift between the network of QS and $\mathrm{CH}$ and between the internetwork of QS and CH. From this panel, we can see that the lines in the network of $\mathrm{CH}$ are systematiclly more blueshifted than those of QS. In CH, a large portion of the materials related to the blueshift in the network are injected into the solar wind along the open field line, and there is a significant reduction in returning mass from the corona there than in QS (McIntosh et al. 2011). That there is less returning mass in $\mathrm{CH}$ is probably why $\mathrm{CH}$ network is more blueshifted than QS. In the internetwork regions we can see that, when the temperature is lower than $\log (T / \mathrm{K})=5.3$, there is no significant difference between the Doppler shift in QS and in CH. In the hotter lines, the difference shows up but has lower absolute values than those in network. The difference in the shift at the lower altitude in internetwork tells us that in the chromosphere and lower TR, the internetwork regions of QS and CH largely have the same characteristics. The difference in the shift at the higher altitude may be related to the expansion of the magnetic field lines there that can cause the contamination by the network signals in the internetwork regions.

The bottom righthand panel shows the Doppler shift difference between network and internetwork in QS and the networkinternetwork difference in $\mathrm{CH}$. We can see that in QS the network is $1-5 \mathrm{~km} \mathrm{~s}^{-1}$ more redshifted than internetwork, but the difference in $\mathrm{CH}$ is much smaller. This small difference may be due to the stronger expansion of the open field lines from network to internetwork in CH than in QS (Tian et al. 2008), and it could also be caused by the low signal-to-noise and high uncertainty in the $\mathrm{CH}$. 
X. Wang et al.: Temperature dependence of UV line parameters
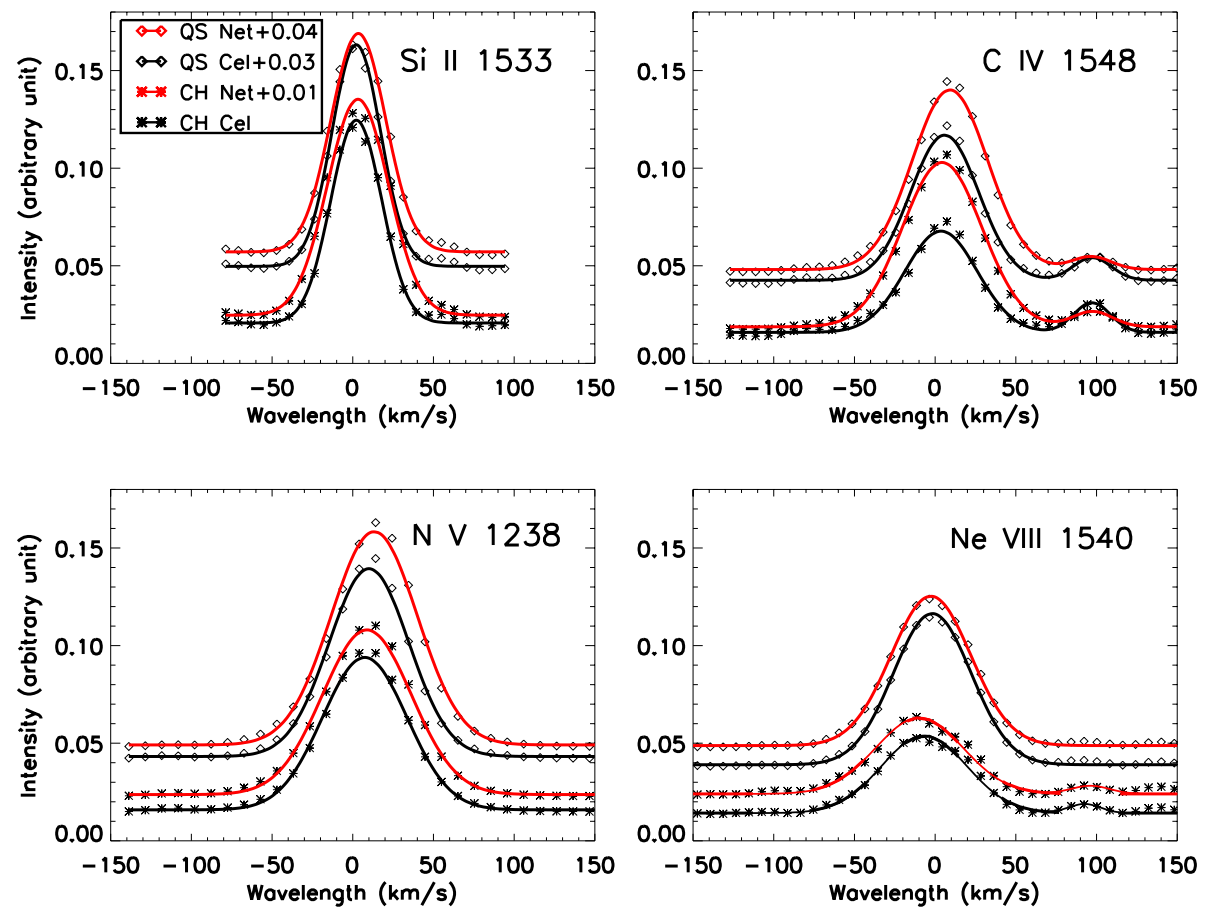

Fig. 3. Normalized average line profiles of Si II, $\mathrm{C}$ IV, $\mathrm{N} \mathrm{v}$, and Ne VIII. The observed spectra in QS and $\mathrm{CH}$ are shown as the diamonds and asterisks. The solid lines are single-Gaussian (lefthand panels) or multi-Gaussian (righthand panels) fitting results in network (red) and internetwork regions (black). We shift the profiles in QS network, QS internetwork and CH network regions in the $y$-axis by $0.04,0.03$, and 0.01 , respectively, to avoid the overlap between the different spectra.
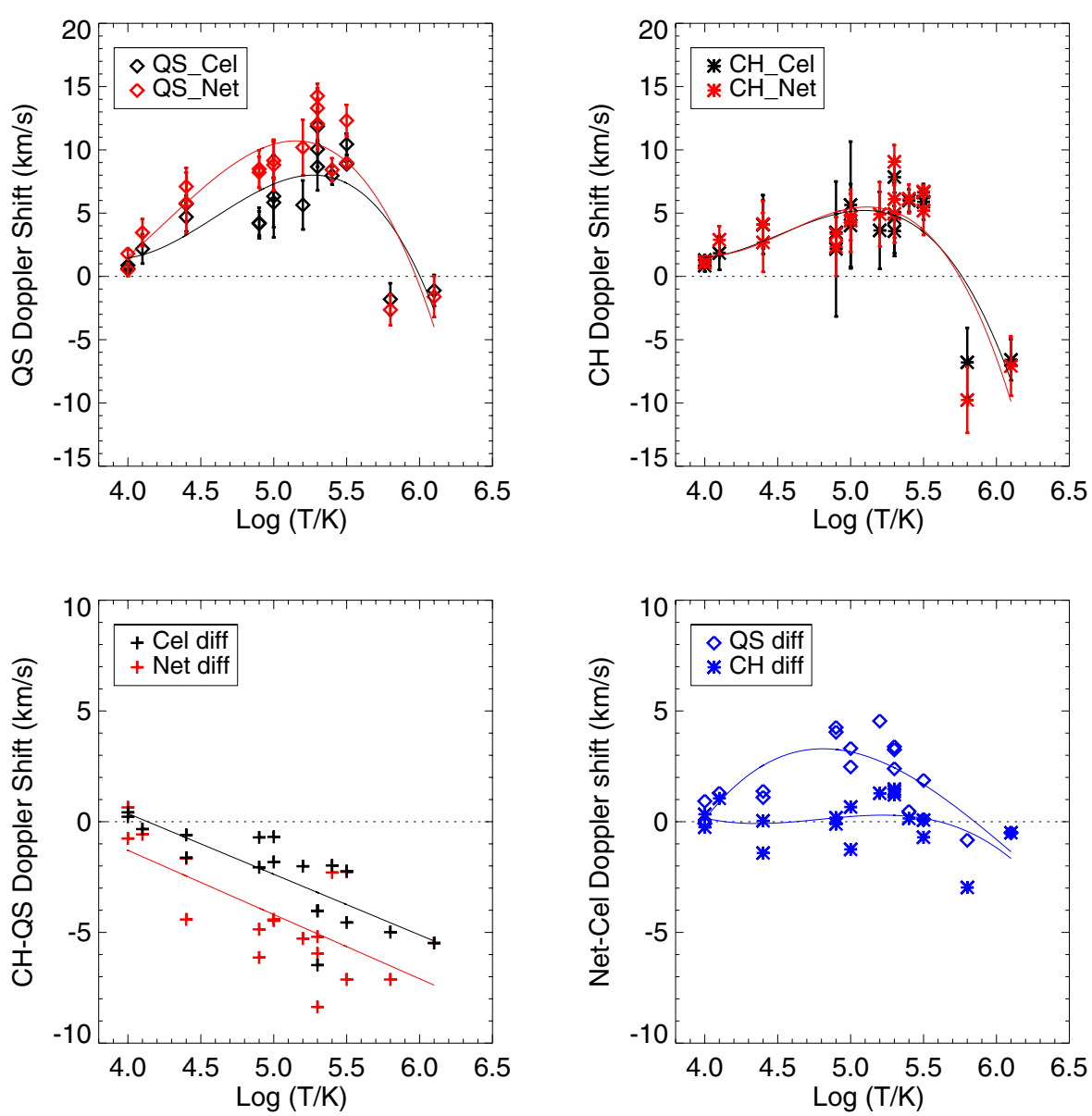

Fig. 4. Average Doppler shifts of various ions measured from UV lines as a function of the formation temperature in network (red) and internetwork (dark) regions of QS (diamonds) and $\mathrm{CH}$ (asterisks). A) QS; B) $\mathrm{CH}$; C) difference of the Doppler shift between the $\mathrm{CH}$ and QS; D) difference in the Doppler shift between network and internetwork regions. The error bars present the errors of the fits. The solid lines in every panel are the polynomial fitting results of those scatter points.

\subsection{Average non-thermal widths}

Figure 5 shows the average non-thermal widths of various ions as a function of the formation temperature in network and internetwork regions of QS and $\mathrm{CH}$ in the same manner as in Fig. 4 (see also Table 2). It can be seen from Fig. 5 that the non-thermal widths peak at $\log (T / \mathrm{K}) \approx 5.3$ and decrease toward the lower and higher temperature. This result is consistent with previous studies (Chae et al. 1998a; Teriaca et al. 1999). One interesting thing is that the peaks of the widths show up nearly at the same temperature as the peak of the average Doppler shift (see Fig. 4), a result that will be discussed in detail below. From the bottom 

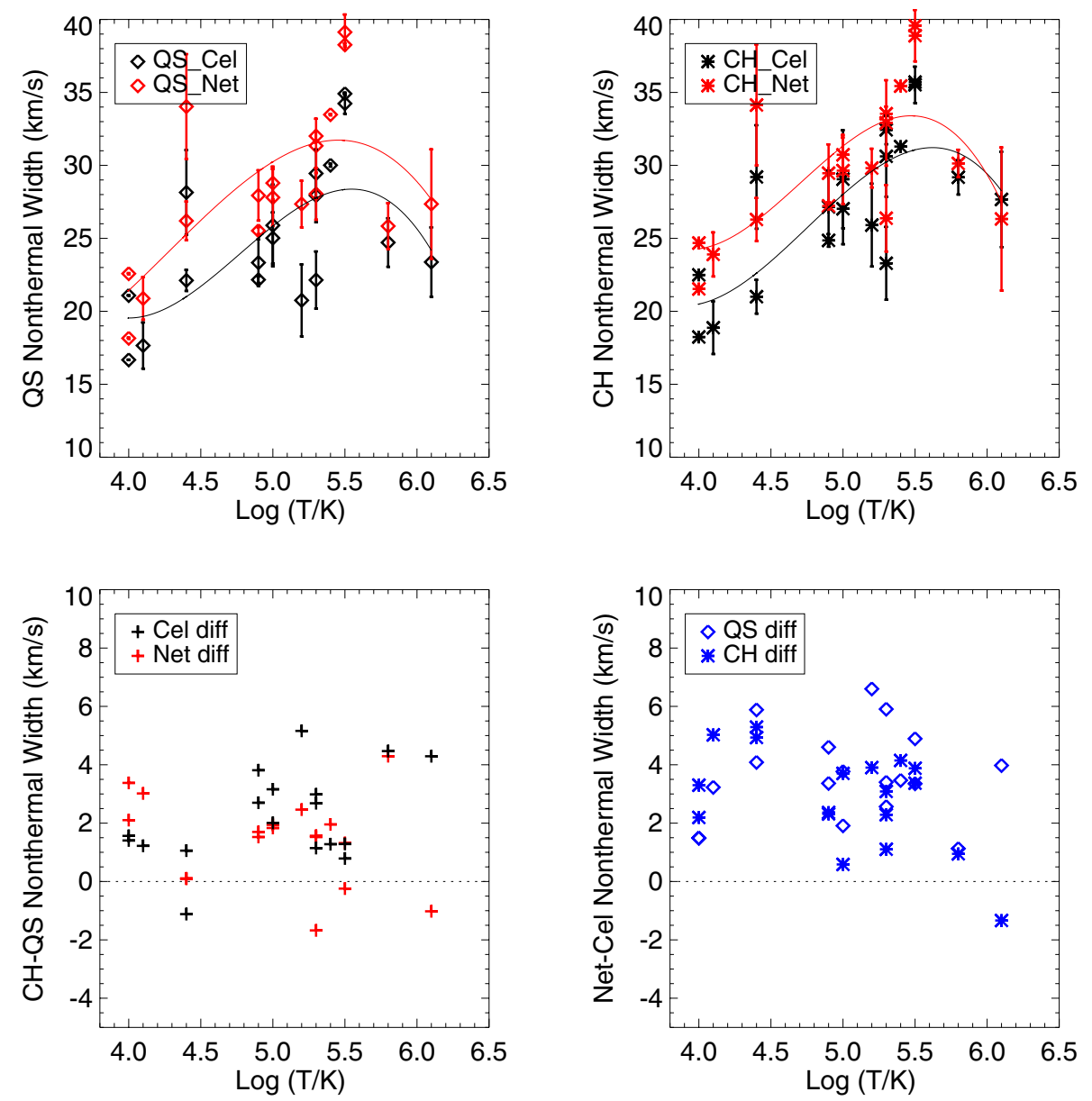

Fig. 5. Average non-thermal widths of various ions as a function of the formation temperature in network and internetwork regions of QS and $\mathrm{CH}$ in the same manner as in Fig. 4.

panels, we know that the non-thermal width is greater in $\mathrm{CH}$ than in QS in both network and internetwork and also greater in the network than in the internetwork of both QS and $\mathrm{CH}$, since almost all the scattered points are located above the horizontal zero lines. The enhancement of the line width in the network reminds us once again that the network and internetwork regions are probably dominated by different physical processes, and we need to separate the network and internetwork when discussing the dynamics and physical properties of the solar atmosphere.

\section{Two simple scenarios}

\subsection{Three-component scenario}

The results presented above are important to help us understand more about the persistent redshifts in TR and the complex process of the mass cycle between the chromosphere and corona. McIntosh et al. (2012) proposes that the ensemble UV emission of the solar atmosphere comes from three parts: 1) a rapid (50-100 $\mathrm{km} \mathrm{s}^{-1}$ ), weak heating upflow at the beginning of the mass cycle generated in the lower atmosphere, and this part is visible in both cool (Si VII) and hot (Fe XII) emission passbands. 2) A slow (10-20 $\mathrm{km} \mathrm{s}^{-1}$ ), cooling/draining downflow at the end of the cycle, and this part can be just observed in cool lines. 3) a nearly static background emission. Figure 5 of McIntosh et al. (2012) shows a cartoon to illustrate this mass-cycling process.

Accordingly, a three-component toy model is built here to see whether the combination of the three components is able to produce our observational curves for the variation in line parameters as a function of the formation temperature in QS and $\mathrm{CH}$.
Since the physical processes in the internetwork are too complex, we just focus on the network regions. We now construct artificial line profiles for 12 lines with different formation temperatures ranging from $10^{4} \mathrm{~K}$ to $1.2 \times 10^{6} \mathrm{~K}$. Each of the artificial profiles is built with three emission components, including a background component, a redshift component (cooling downflow), and a blueshift component (heating upflow). Panels $\mathrm{A}-\mathrm{C}$ of Fig. 6 show the values of the three line parameters for each component of 12 lines, and the values are plotted as a function of the line formation temperature. They are set according to previous works (e.g., De Pontieu et al. 2009; De Pontieu \& McIntosh 2010; McIntosh \& De Pontieu 2009a,b; McIntosh et al. 2011,2012; Tian et al. 2011a,b) and based on the following assumptions.

1) Intensity: the intensity of the background is set as a constant value of 100 , and the intensities we mention in the following all refer to the relative intensities to the background component. The intensities of cooling downflow and heating upflow are shown in panels $\mathrm{A}$ and $\mathrm{C}$ of Fig. 6, respectively. a) Downflow: at the height with very high temperature (corona), the downflow is certainly very weak since the downflow results from the cooling process. At the height with low temperature (chromosphere), both the strong emission background and the viscosity of the high-density chromosphere can reduce the relative intensity of the downflow. Therefore, we expect that the profile of the downflow intensity peaks at a certain TR temperature. Because of the large amount of open field lines and few closed loops, the intensity of the downflow in $\mathrm{CH}$ is set to be half the intensity in QS. b) Upflow: based on the statistical results of 

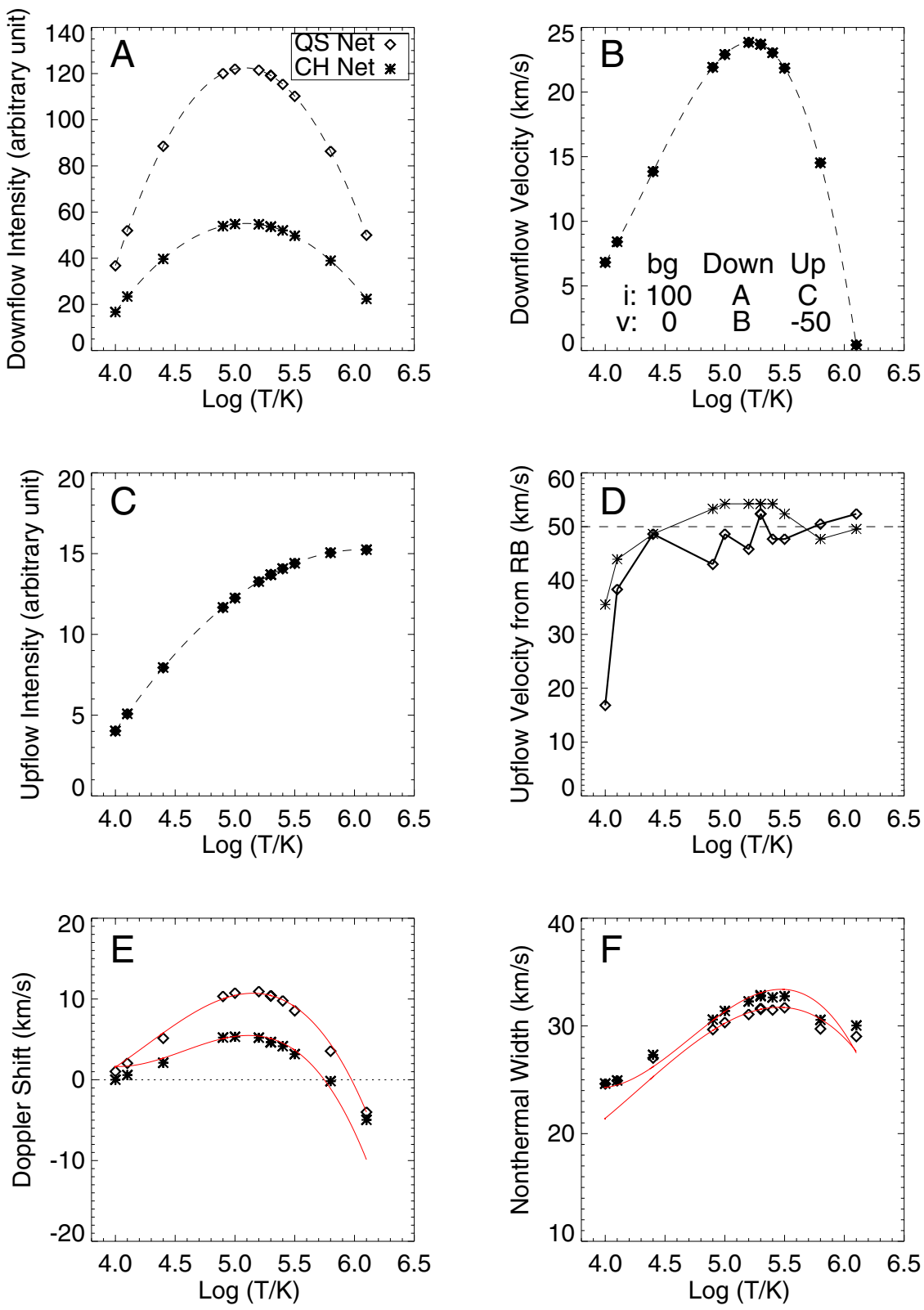

Fig. 6. Input line parameters of the three emission components for the artificial line profiles and the results of RB asymmetry analysis and the output line parameters derived from single-Gaussian fit on the artificial profiles versus formation temperature, with diamonds for QS network and asterisks for $\mathrm{CH}$ network. A) Intensity of the downflow (cooling) component; B) velocity of the downflow component; C) intensity of the upflow (heating) component; D) upflow velocity derived from RBp asymmetry analysis; E)-F) Doppler-shift velocity and non-thermal line width determined from singleGaussian fit. The dashed lines in A), B), and C) panels are the second-order polynomial fit of the input parameters. The red solid lines in panels E) and F) are adopted from the observational results discussed above (Figs. 4 and 5). In panel B), the other input parameters are noted. The background component, redshift component (cooling downflow), and blueshift component (heating upflow) are marked as "bg", "down", and "up", respectively. The intensity and Doppler velocity are marked by "i" and "v", respectively.
Tian et al. (2011b) and Tian et al. (2012), the intensity of the upflow is set to be $5 \%$ to $15 \%$ of the background intensity.

2) Doppler velocity: the background is assumed to be at rest. a) Downflow: as the temperature decreases, the velocity of the downflow (shown in panel B of Fig. 6) increases from $0 \mathrm{~km} \mathrm{~s}^{-1}$ to a maximum value (tens of $\mathrm{km} \mathrm{s}^{-1}$ ) at first, and then decreases to a few $\mathrm{km} \mathrm{s}^{-1}$. The acceleration of the downflow at the beginning is caused by the gravitational effect, as the plasma begins to cool down and begins to fall downward. The deceleration of the downflow at the layer with lower temperature is due to the stronger resistance (e.g., friction) of the dense chromospheric and TR background during its falling down process. b) Upflow: Previous investigations have pointed out that at the loop footpoints of AR, the speed of the upflow component is $50-150 \mathrm{~km} \mathrm{~s}^{-1}$ and is roughly constant across a temperature range of 100000 to several million degrees (De Pontieu et al. 2009; McIntosh \& De Pontieu 2009a,b; Tian et al. 2011a,b). Since the upflow is slower in $\mathrm{QS}$ and $\mathrm{CH}$ than in $\mathrm{AR}$, we set the upflow velocity as the lower limit of $50 \mathrm{~km} \mathrm{~s}^{-1}$ for all the ions here.

3) Line width: the non-thermal width of the line profiles is set as $23 \mathrm{~km} \mathrm{~s}^{-1}$ according to our observational results shown in Fig. 5. The instrumental broadening (Doppler width) of SUMER is quoted from Chae et al. (1998a) as $0.06 \AA$. The total line width is given by the combination of the nonthermal width, instrumental broadening, and thermal width. Here the line widths of the three components are set to be the same. Our choice of the same width for each component is a reasonable assumption. For instance, Tian et al. (2011b) applied the RB-asymmetry analysis to both artificial spectra and spectra observed by the Extreme-ultraviolet Imaging Spectrometer (EIS) onboard Hinode, and demonstrated that the line widths of different emission components are comparable. The assumption of same line width has also been used by De Pontieu \& McIntosh (2010). Although these studies are focused on observations at active region boundaries, we 

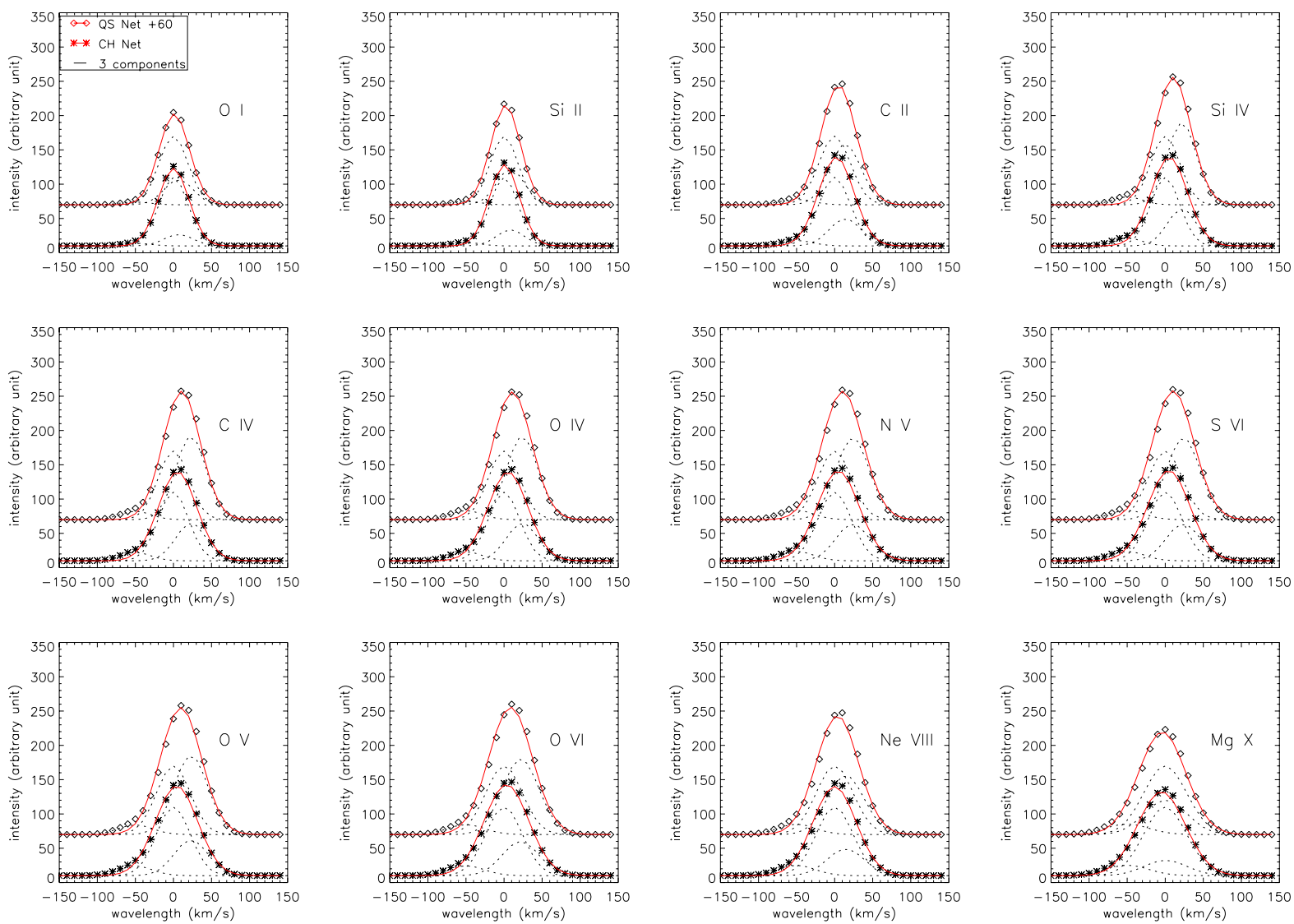

Fig. 7. Artificial line profiles of ions with different formation temperature ranging from $\log (T / \mathrm{K})=4.0$ to $\log (T / \mathrm{K})=6.1$. The three emission components are shown as dotted lines, and the total profiles are shown as diamonds (QS network) and asterisks (CH network). Red lines are the single-Gaussian fitting profiles. The QS network profiles are shifted in the $y$-axis by 60 to avoid the overlap with $\mathrm{CH}$ profiles.

cannot think of a reason why this is not the case in QS. Bryans et al. (2010) from a different group also used this assumption in their multi-Gaussian algorithm.

The artificial line profiles of twelve lines with the line parameters described above are shown in Fig. 7. The dotted lines are the three Gaussian components. And the diamonds and asterisks are the total emission of them in QS network and in $\mathrm{CH}$ network, respectively. The solid red lines are the singleGaussian fit to the ensemble spectra, just like what we have done for the real observed line profiles. The spectra in QS are shifted in the $y$-axis. Panel D of Fig. 6 represents the upflow velocity derived from the $R B_{\mathrm{p}}$ asymmetry analysis (De Pontieu et al. 2009; Martínez-Sykora et al. 2011; Tian et al. 2011b). The single-Gaussian fit results of the Doppler velocity and the nonthermal width are shown in the panel E and panel F of Fig. 6, again with diamonds for QS and asterisks for $\mathrm{CH}$. The four red lines are copied from the red lines in the top two panels of Figs. 4 and 5, which account for the observational variation of the Doppler shift and non-thermal width as a function of temperature.

The close agreement between the scatter points (toy model results) and the red lines (observational results) in panel $\mathrm{E}$ and $\mathrm{F}$ of Fig. 6 implies that the existence and the combination of the three components can self-consistently explain the observational curves, and that the temperature dependence of Doppler shifts and line widths might be caused by the different relative contributions of the three components at different temperatures. Even though it is just a simple toy model, where more work is needed to define the parameters better, we can still see that such a combination of the three components is able to explain all the previous results of the temperature dependence of the Doppler shift and non-thermal width determined solely from a singleGaussian fit.

\subsection{Two-component scenario}

By applying a completely free double-Gaussian fit to the spectral profiles of several TR lines in the network of QS, Peter (2000, 2001) found that the UV SUMER spectra could be decomposed to a bright core component related to the small loops and a faint wide "pedestal" component emitted from the coronal funnels (rooted in the supergranular network). Furthermore, Peter (2010) used the data from EIS onboard Hinode and finds that in the core of an active region, the spectra of the Fe XV $284 \AA$ line are best fit by a narrow line core and a broad minor component. The latter contributes $10 \%$ to $20 \%$ to the total emission, is about twice as broad as the former, and has strong blueshifts of about $50 \mathrm{~km} \mathrm{~s}^{-1}$. In the following, a two-component scenario based on the observations of Peter $(2000,2001,2010)$ is discussed. In this scenario, the total emission line profiles are the sum of a bright core component (CC) and a faint wide tail component (TC). Since only the network regions in the QS conditions were investigated in Peter (2001), and we do not have any observational information about a two-component scenario in $\mathrm{CH}$, we just focus on the network regions of QS.

In Fig. 8, panels $\mathrm{A}, \mathrm{B}$, and $\mathrm{C}$ show the line parameters (relative intensity, velocity, and non-thermal line width) of TC and $\mathrm{CC}$ as a function of temperature. The intensity of $\mathrm{CC}$ is 
X. Wang et al.: Temperature dependence of UV line parameters
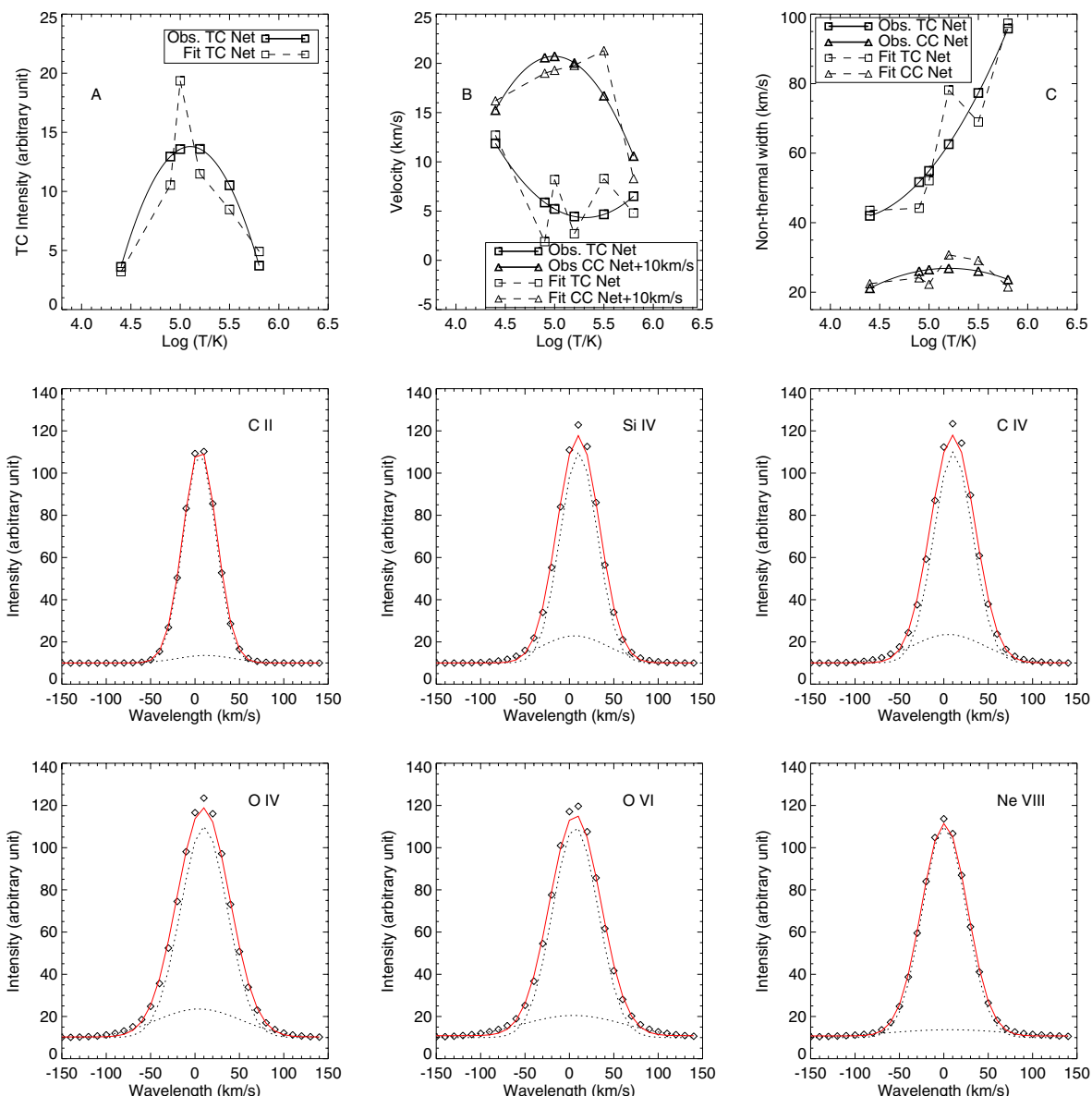

Fig. 8. Six artificial line profiles and the line parameters of the two components. The first row: the variation in the line parameters of the narrow TC (squares) and broad CC (triangles) as a function of formation temperature in QS network condition, with panels A), B), and C) for relative intensity, Doppler velocity, and non-thermal line width, respectively. The dashed lines show the observational results of Peter (2001), and the solid lines show the second-order polynomial fit to them. In panel B), the Doppler velocity of CC is shifted in the $y$-axis by $10 \mathrm{~km} \mathrm{~s}{ }^{-1}$ to avoid the overlap with TC velocity. The bottom two rows: the artificial line profiles of C II, Si IV, C IV, O IV, O VI, and Ne VIII. They are built according to the line parameters as shown in solid lines in the top three panels. The dotted lines represent the profiles of TC and CC, and the diamonds are the sum of them. The red solid lines show the single-Gaussian fits to the total emission profiles.
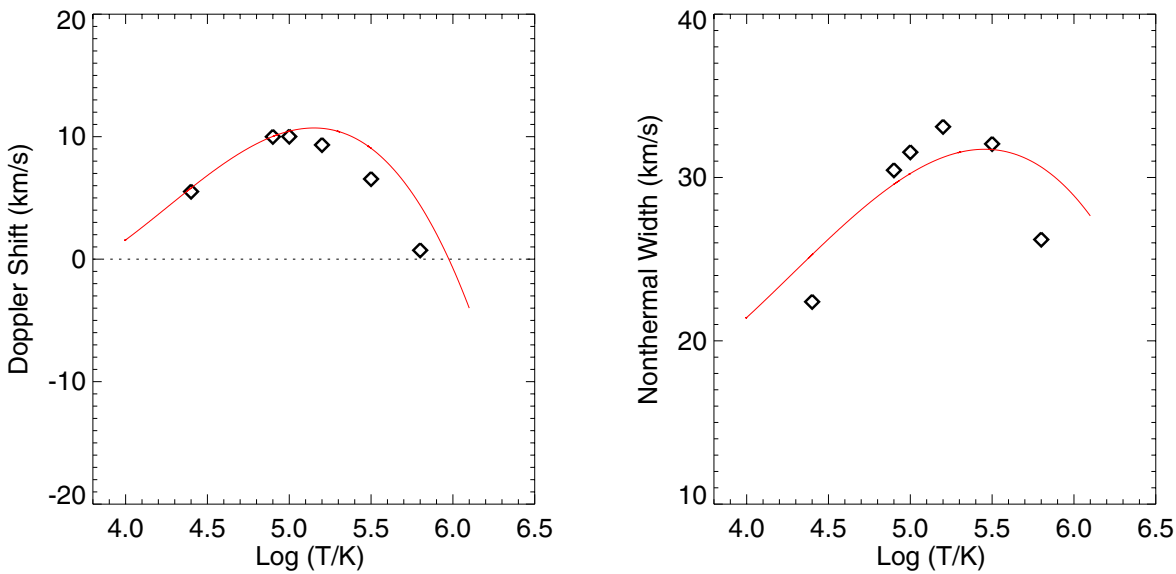

Fig. 9. Two-component model results. The diamonds represent the Doppler shift and nonthermal line width derived from the singleGaussian fit to the artificial profiles shown in Fig. 8. The red solid lines, adopted from Figs. 4 and 5, show our observational results of the temperature dependence of the line parameters in QS network.

set as a constant value of 100 , so the TC intensity as shown in panel A is the relative intensity to CC. The Doppler shift velocities of TC and the single-Gaussian fit of the line profiles (SG) listed in Table 1 of Peter (2001) are the relative velocities to CC, i.e., $V_{\mathrm{TC}-\mathrm{CC}}$ and $V_{\mathrm{SG}-\mathrm{CC}}$. To get the absolute velocities, we use the velocities in the network regions of QS in the top lefthand panel of Fig. 4 as the velocity of $\mathrm{SG}, V_{\mathrm{SG}}$. Then the velocities of TC and $\mathrm{CC}$ can be calculated from $V_{\mathrm{CC}}=V_{\mathrm{SG}}-V_{\mathrm{SG}-\mathrm{CC}}$ and
$V_{\mathrm{TC}}=V_{\mathrm{CC}}-V_{\mathrm{TC}-\mathrm{CC}}$. The bottom two rows of Fig. 8 show the artificial line profiles of the six ions.

From Fig. 9, we can see that the Doppler shift and nonthermal line width derived from a single-Gaussian fit to the artificial line profiles are consistent with our previous observational results in the network regions of QS (the red line in top lefthand panels of Figs. 4 and 5) very well, which indicates the agreement between the two-component scenario and observations. 
While it does provide a good fit to existing data, the twocomponent scenario of Peter $(2000,2001)$ has a very different physical interpretation from the three-component scenario presented above. The two-component scenario can be interpreted as composed of a slowly falling plasma component and a faint "pedestal" component, where the latter has a strong broadening (e.g., due to high frequency plasma waves) and was originally interpreted as originating in "coronal funnels". In contrast, the core of the three-component scenario is not moving, and there is a high velocity upflow component and a moderately downward flow component that combine in a resolution element; indeed, we cannot discount that the latter wing components would not combine to form a broad pedestal for the core of the line and visually match the analysis of Peter $(2000,2001)$. It is still the case, however, that one must reconcile the observed high speeds of Type II spicules in the magnetic network and active regions (De Pontieu et al. 2007, 2009), their relationship with line profile asymmetries (McIntosh \& De Pontieu 2009a,b), and the apparent temperature dependence of the downflow component strength (McIntosh et al. 2012) in building a comprehensive picture of mass transport in the solar atmosphere. Unfortunately, it is clear that the spatial/spectral/temporal resolution of the SUMER or EIS observations are not sufficient to address the complexity of either case.

New instrumentation is needed to distinguish between those scenarios that rely on spatially unresolved components of the plasma with different flow structures. Besides a higher spatial resolution to better differentiate between the components spatially, an improved spectral resolution is pivotal. With more data points across the line profile and a narrower instrumental broadening observationally, one might be able to decide which of these scenarios provides a better fit. IRIS will provide all these improvements: a factor of 4 in spatial resolution and a factor of 3 in spectral resolution as compared to SUMER, as well as the smaller instrumental width.

In the IRIS range, there are about seven spectral lines that can be used to investigate the temperature dependence of average Doppler shift and line width. They are C II $1335.71 \AA$, Fe XII $1349.38 \AA$ A, O I $1355.60 \AA$ A, Ni II $1381.30 \AA$, O IV $1401.16 \AA$, Si IV $1402.77 \AA$, and Fe II 1405.61 $\AA$. These lines are mostly optically thin and strong. They are also clean or can be easily separated from the blend. Nevertheless, since IRIS is designed to investigate mainly the solar chromosphere and transition region, the formation temperatures of these lines are lower than $10^{5} \mathrm{~K}$, except for O IV 1401.16 with $\log (T / \mathrm{K}) \approx 5.2$ and Fe XII $1349.38 \AA$ with $\log (T / K) \approx 6.1$. The IRIS lines are undoubtedly better for the components decomposition, therefore, but the sampling at the high temperature will be not as good as for the SUMER lines.

\section{Conclusions}

We have investigated the Doppler shift and non-thermal line width of various lines with formation temperatures ranging from $10^{4} \mathrm{~K}$ to $1.2 \times 10^{6} \mathrm{~K}$ in the network and internetwork of QS and $\mathrm{CH}$ of the solar atmosphere. The values of the Doppler shift magnitude and the non-thermal line width are found to be larger in network than in internetwork regions, which indicates that the network and internetwork regions are dominated by different physical processes. It is necessary to separate the network and internetwork when discussing the dynamics and physical properties of the solar atmosphere.

We also find that the line profiles in the network of $\mathrm{CH}$ are broader and more blueshifted than in the network of QS nearly in the whole range of the temperature, while the difference in the Doppler shifts in the internetwork between them is smaller, especially when $\log (T / \mathrm{K})$ is lower than 5.3. In the network, a large portion of the $\mathrm{CH}$ material related to the blueshift is injected into the solar wind along the open field line, and there is less returning mass from the corona there than in QS. This results in the network being more blueshifted in the $\mathrm{CH}$ than in the QS. Moreover, since no Doppler shift difference is found between the $\mathrm{CH}$ and QS internetwork regions at the lower solar atmosphere with $\log (T / \mathrm{K})<5.3$, it is reasonable to speculate that the physical processes of the QS and $\mathrm{CH}$ internetwork are likely to be the same, and the difference in the high TR and corona of the shift between network and internetwork is due to the expansion of the magnetic field lines at the higher atmosphere that can lead to contamination by the network signals in the internetwork regions.

To understand the temperature dependence of the line profiles, a simple three-component toy model has been built in the network regions of $\mathrm{QS}$ and $\mathrm{CH}$ on the basis of previous studies and some assumptions. The three-component scenario was proposed by McIntosh et al. (2012). In this scenario, the UV emission line profiles from the solar atmosphere are suggested to consist of three Gaussian components: a rapid, weak, and heating upflow; a slow, cooling/draining downflow; and a nearly static background emission. The agreement between the single-Gaussian fit results of our toy model profiles and the observational curves tells us that the temperature dependence of the line parameters might be caused by the different relative contributions of the three components at different temperatures. The superposition of heating upflows and cooling downflows on the background emission in the chromosphere-coronal mass cycle modulates the line shift and non-thermal line width of the singleGaussian fit, and is able to explain their variation with formation temperature. It also reminds us that a single-Gaussian fit to emission profiles would not be sufficient to describe the complex underlying physics of the solar atmosphere. The RB asymmetry analysis, which is based on the assumption of two components, should also be used more carefully due to the various flow systems in the ensemble emission, especially at the TR temperature as mentioned in McIntosh et al. (2012).

Likewise, Peter $(2001,2010)$ has suggested a twocomponent scenario, which includes a bright core component and a faint wide tail component. Unfortunately, with current spectrographs, it is difficult for us to decompose the UV emission line profiles to either three or two components without any ambiguity. At this stage, IRIS with high spectral and spatial resolution and small instrumental line width is highly desirable. Hopefully with IRIS we can get the chance to know more details and the real physical processes of the solar atmosphere.

Acknowledgements. X.W. is supported by China Scholarship Council for her stay at HAO. X.W. is also supported by NSFC under contract Nos. 41174148 , 40931055, 41231069, and 41222032. S.W.M. and H.T. are supported by NSF ATM-0925177 and NASA NNG06GC89G. SWM is partially supported by IRIS mission grant NNG08FD61C. H.T. is also supported by the ASP Postdoctoral Fellow-ship Program of NCAR. L.D.X. is supported by the National Natural Science Foundation of China (NSFC) under contract No. 40974105. The SUMER project is financially supported by DLR, CNES, NASA, and the ESA PRODEX program (Swiss contribution). SUMER is part of SOHO, the Solar and Heliospheric Observatory, of ESA and NASA.

\section{References}

Aiouaz, T., Peter, H., \& Lemaire, P. 2005, 435, 713

Brekke, P., Hassler, D. M., \& Wilhelm, K. 1997, Sol. Phys., 175, 349

Brooks, D. H., Warren, H. P., \& Young, P. R. 2011, ApJ, 730, 85 
Bryans, P., Young, P. R., \& Doschek, G. A. 2010, ApJ, 715, 1012 Brynildsen, N., Brekke, P., Fredvik, T., et al. 1998, Sol. Phys., 181, 23 Chae, J., Schühle, U., \& Lemaire, P. 1998a, ApJ, 505, 957

Chae, J., Yun, H. S., \& Poland, A. I. 1998b, ApJ, 114, 15

Curdt, W., Brekke, P., Feldman, U., et al. 2001, A\&A, 375, 591

Curdt, W., Tian, H., Dwivedi, B. N., \& Marsch, E. 2008, A\&A, 491, L13

Dadashi, N., Teriaca, L., \& Solanki, K. 2011, A\&A, 534, A90

Dammasch, I. E., Wilhelm, K., Curdt, W., \& Hassler, D. M. 1999, A\&A, 346, 285

Dammasch, I. E., Curdt, W., Dwivedi, B. N., \& Parenti, S. 2008, Ann. Geophys., 26,2955

Davey, A. R., McIntosh, S. W., \& Hassler, D. M. 1990, ApJS, 165, 386

Deubner, F.-L., \& Fleck, B. 1990, A\&A, 228, 506

De Pontieu, B., \& McIntosh, S. W. 2010, ApJ, 722, 1013

De Pontieu, B., McIntosh, S. W., Hansteen, V. H., et al. 2007, PASJ, 59, S655

De Pontieu, B., McIntosh, S. W., Hansteen, V. H., \& Schrijver, C. J. 2009, ApJ, 701, L1

Doschek, G. A., Bohlin, J. D., \& Feldman, U. 1976, ApJ, 205, L177

Feldman, U., Doschek, G. A., \& Cohen, L. 1982, ApJ, 255, 325

Hansteen, V. H. 1993, ApJ, 402, 741

Hansteen, V. H., Hara, H., De Pontieu, B., \& Carlsson, M. 2010, ApJ, 718, 1070

Hassler, D. M., Dammasch, I. E., Lemaire, P., et al. 1999, Science, 283, 810

He, J.-S., Tu, C.-Y., \& Marsch, E. 2008, Sol. Phys., 250, 147

Kaufman, V., \& Martin, W. C. 1989, J. Opt. Soc. Am., 6, 1769

Kelly, R. L. 1987, Atomic and ionic spectrum lines below 2000 Angstroms Hydrogen through krypton (New York: AIP, American Chemical Society and the National Bureau of Standards), 16

Lemaire, P., Wilhelm, K., Curdt, W., et al. 1997, Sol. Phys., 170, 105

Lemaire, P., Bocchialini, K., Aletti, V., Hassler, D., \& Wilhelm, K. 1999, Sol. Phys., 87, 249

Martínez-Sykora, J., De Pontieu, B., Hansteen, V., \& McIntosh, S. W. 2011, ApJ, 732,84

Mazzotta, P., Mazzitelli, G., Colafrancesco, S., \& Vittorio, N. 1998, A\&AS, 133, 403
McIntosh, S. W. \& De Pontieu, B. 2009a, ApJ, 706, L80 McIntosh, S. W., \& De Pontieu, B. 2009b, ApJ, 707, 524

McIntosh, S. W., Davey, A. R., Hassler, D. M., et al. 2007, ApJ, 654, 650

McIntosh, S. W., Innes, D. E., De Pontieu, B., et al. 2010, A\&A, 510, A2

McIntosh, S. W., Leamon, R. J., \& De Pontieu, B. 2011, ApJ, 727, 7

McIntosh, S. W., Tian, H., Sechler, M., \& De Pontieu, B. 2012, ApJ, 749, 60

Pneuman, G. W., \& Kopp, R. A. 1978, Sol. Phys., 57, 49

Peter, H. 1999, ApJ, 516, 490

Peter, H. 2000, A\&A, 360, 761

Peter, H. 2001, A\&A, 374, 1108

Peter, H. 2010, A\&A, 521, A51

Peter, H., \& Judge, P. G. 1999, ApJ, 522, 1148

Peter, H., Gudiksen, B., \& Nordlund, A. 2004, ApJ, 617, 85

Peter, H., Gudiksen, B., \& Nordlund, Å. 2006, ApJ, 638, 1086

Stuki, K., Solanki, S. K., Schühle, U., et al. 2000, Ann. Geophys., 363, 1145

Teriaca, L., Banerjee, D., \& Doyle, J. G. 1999, A\&A, 349, 636

Tian, H., Marsch, E., Tu, C.-Y., et al. 2008, A\&A, 482, 267

Tian, H., Tu, C.-Y., Marsch, E., He, J.-S., \& Kamio, S. 2010, ApJ, 709, L88

Tian, H., McIntosh, S. W., \& De Pontieu, B. 2011a, ApJ, 727, L37

Tian, H., McIntosh, S. W., De Pontieu, B., et al. 2011b, ApJ, 738, 18

Tian, H., McIntosh, S. W., Xia, L.-D, et al. 2012, ApJ, 748, 106

Tu, C.-Y., Zhou, C., Marsch, E., et al. 2005a, Science, 308, 519

Tu, C.-Y., Zhou, C., Marsch, E., et al. 2005b, ApJ, 624, L133

Wiegelmann, T., Xia, L.-D., \& Marsch, E. 2005, A\&A, 432, L1

Wilhelm, K., Curdt, W., Marsch, E., et al. 1995, Sol. Phys., 162, 189

Wilhelm, K., Lemaire, P., Curdt, W., et al. 1997, Sol. Phys., 170, 75

Wilhelm, K., Dammasch, I. E., Marsch, E., \& Hassler, D. M. 2000, A\&A, 353, 749

Xia, L.-D., Marsch, E., \& Curdt, W. 2003, A\&A, 399, L5

Xia, L.-D., Marsch, E., \& Wilhelm, K. 2004, A\&A, 424, 1025

Yang, S. H., Zhang, J., Jin, C. L., et al. 2009, A\&A, 501, 745

Zhang, J., Wang, J., Deng, Y., \& Wang, H. 1999, Sol. Phys., 188, 47 